\title{
Anwendungsfälle und Konzeptentwicklung eines Pedelec-Prüfstandes
}

\author{
T. Weickert ${ }^{1}$, W. Götzmann ${ }^{2}$
}

\section{Zusammenfassung}

Die Fakultät für Elektrotechnik der Hochschule Mannheim hat ein Leitprojekt Elektromobilität initiiert, in dessen Rahmen u.a. ein Prüfstand für elektrisch unterstützte Fahrräder, die sogenannten Pedelecs, entwickelt werden soll. Mit dem Prüfstand und seiner Entwicklung sollen zum einen Studierende an das Thema Elektromobilität herangeführt werden, andererseits sollen den Studierenden praxisnahe Arbeiten begleitend zu Vorlesungen ermöglicht werden. Dabei soll der Prüfstand aber auch tatsächliche Anforderungen der Zweirad-Industrie adressieren.

Der Posterbeitrag trägt zunächst Anwendungsfälle für Pedelec-Prüfstände zusammen. Darauf aufbauend werden Anforderungen für den Prüfstandsaufbau sowie benötigte Betriebsmodi abgeleitet.

\section{Stichwörter}

Pedelec, Elektrofahrräder, Prüfstand

\section{Einleitung und Motivation}

Durch den sich immer rascher vollziehenden Klimawandel ist Elektromobilität bereits in aller Munde und wird in den kommenden Jahren weiter an Bedeutung gewinnen. Während die Elektromobilität im Automobilbereich noch vergleichsweise am Anfang dieser Entwicklung steht, haben elektrisch unterstützte Zweiräder (EPACs - electrically power assisted cycles) im Jahr 2020 bereits einen Marktanteil von fast $40 \%$ erreicht. [2]

Aus diesen Gründen hat die Fakultät für Elektrotechnik der Hochschule Mannheim ein Leitprojekt Elektromobilität initiiert, in dessen Rahmen u.a. ein Prüfstand für elektrisch unterstützte Fahrräder, die sogenannten Pedelecs, entwickelt werden soll. Mit dem Prüfstand und seiner Entwicklung sollen zum einen Studierende an das Thema Elektromobilität herangeführt werden, andererseits sollen den Studierenden praxisnahe Arbeiten begleitend zu Vorlesungen ermöglicht werden. Dabei soll der Prüfstand aber auch tatsächliche Anforderungen der Zweirad-Industrie adressieren.

\section{Anwendungsfälle für einen Pedelec-Prüfstand}

Im Gespräch mit Pedelec-Herstellern sowie durch Vergleich öffentlich verfügbarer Informationen kommerzieller Pedelec-Prüfstände lassen sich eine Reihe von Anwendungsfällen für Pedelec-Prüfstände sammeln:

1 Hochschule Mannheim, Fakultät für Wirtschaftsingenieurwesen

2 Hochschule Mannheim, Fakultät für Elektrotechnik 
- Normprüfung nach DIN EN 15194

- Reichweitentests (z.B. R200 Test des Zweirad-Industrieverbands ZIV)

- Weitere Qualitätssicherung (End-of-Line Tests, Überhitzung, Unterspannung, Rekuperation...)

- Wirkungsgrad- und Effizienzmessungen

Zunächst soll der Pedelec-Prüfstand die Anforderungen der standardisierten Tests aus der Norm DIN EN 15194 [1] sowie des standardisierten Reichweitentest R200 [3] erfüllen.

Die EN 15194 ist sehr umfangreich. Im Rahmen des Projekts wurde entschieden, dass sich der Prüfstand auf die elektrischen Anforderungen konzentrieren soll, insbesondere auf die Prüfung der max. Unterstützungsgeschwindigkeit, die Anfahrunterstützung, das Leistungsmanagement sowie die Messung der Höchstleistung.

Der R200-Test soll im Gegensatz dazu ein vergleichbares Maß für die Reichweite der Batterie liefern, indem in einem standardisierten Test eine Reichweite ermittelt und dann nach bestimmten Normierungsformeln auf eine elektrische Unterstützung von $200 \%$ umgerechnet wird.

\section{Prüfstandsaufbau}

Basierend auf den obigen Anwendungsfällen können Anforderungen für den Aufbau des Prüfstands abgeleitet werden. Beispielsweise muss für den R200-Test die Radaufstandskraft am hinteren Rad 500 $\mathrm{N}$ betragen. Dies bedingt, dass die Bremsrollen, die die auf das Pedelec bremsenden Kräfte darstellen, mit einer vorgebbaren Kraft angedrückt werden können. Eine weitere Anforderung ist, dass sowohl Pedelecs mit Vorderrad- als auch mit Hinterrad- oder Mittelmotor getestet werden können. Daraus resultiert, dass zwei Bremsrollen (vorne und hinten) benötigt werden, die beide angepresst werden müssen.

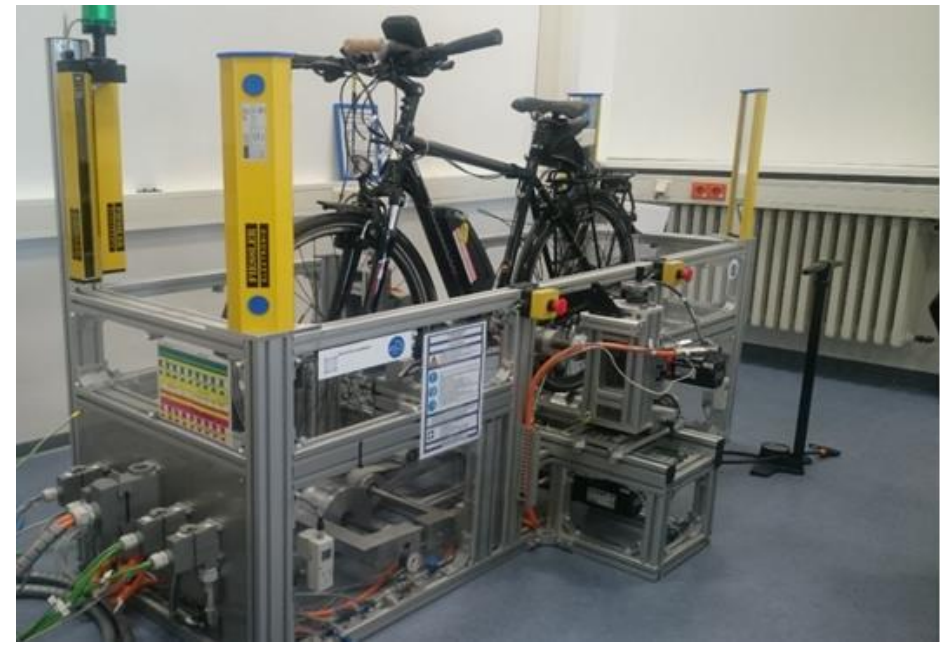

Bild 1: Aktueller Stand des Prüfstandsaufbaus

Der aktuelle Stand des mechanischen Prüfstandsaufbaus, der entsprechend den obigen Zielen und Bedingungen entwickelt wurde, ist in Bild 1 zu sehen.

Pneumatische Zylinder pressen die Bremsrollen an. Die Bremsrollen werden vom Bremsmotor angetrieben, der mit Zahnriemen verbunden ist. Beim Bremsen arbeitet der Motor im Generatorbetrieb. Die entstehende Leistung kann nicht zurückgespeist werden, daher wurde ein Bremswiderstand mit $600 \mathrm{~W}$ Leistung verbaut. Ein Getriebe mit einer Übersetzung von 4:1 zwischen Bremsrollenmotor und Bremsrolle sorgt für eine bessere Ausnutzung des Drehmomentbereichs des Motors.

Der Antrieb der Pedale wird durch zwei Servomotoren realisiert, die den wechselseitigen Fahrertritt simulieren können. An den Motoren sind Getriebe mit Übersetzungsverhältnis 40:1 angeflanscht, um auch hier die Stellbereiche der Motoren besser auszunutzen. Die Motoren sind über eine Rahmenkonstruktion so horizontal und vertikal verstellbar, dass sie auf die Geometrie des getesteten Fahrrads eingestellt werden können.

Um automatisches Schalten zu ermöglichen, wurden zwei Linearmotoren eingebaut, die die Schaltzüge bewegen. 


\section{Betriebsmodi}

Zum Betrieb des Prüfstandes sind verschiedene Modi für die jeweiligen Tests notwendig, wie den R200-Test, die Prüfung der max. elektr. Unterstützung etc.

Darüber hinaus werden aber weitere Betriebsmodi benötigt:

- Fahrrad einmessen: Bestimmung der realen Übersetzungen zwischen Rad und Bremsrolle (inkl. Schlupf, dynamischer Rollradius etc.), der Positionen der Linearmotoren zum Schalten gewünschter Gänge etc.

- Komponententest: Manuelles Ansteuern einzelner Komponenten

- Manueller Fahrradtest: Manuelle Vorgabe von Werten (z.B. Fahrradgeschwindigkeit). Im Gegensatz zum Komponententest sind die Pedalantriebe immer gekoppelt, um den Fahrertritt zu simulieren.

- Fehlerbehandlung: Reaktion auf Fehler des Prüfstandes

Eine vollständige State Machine kann als Zustandsgraph mit allen nötigen Übergängen modelliert werden. Diese ist in Bild 2 zu sehen.

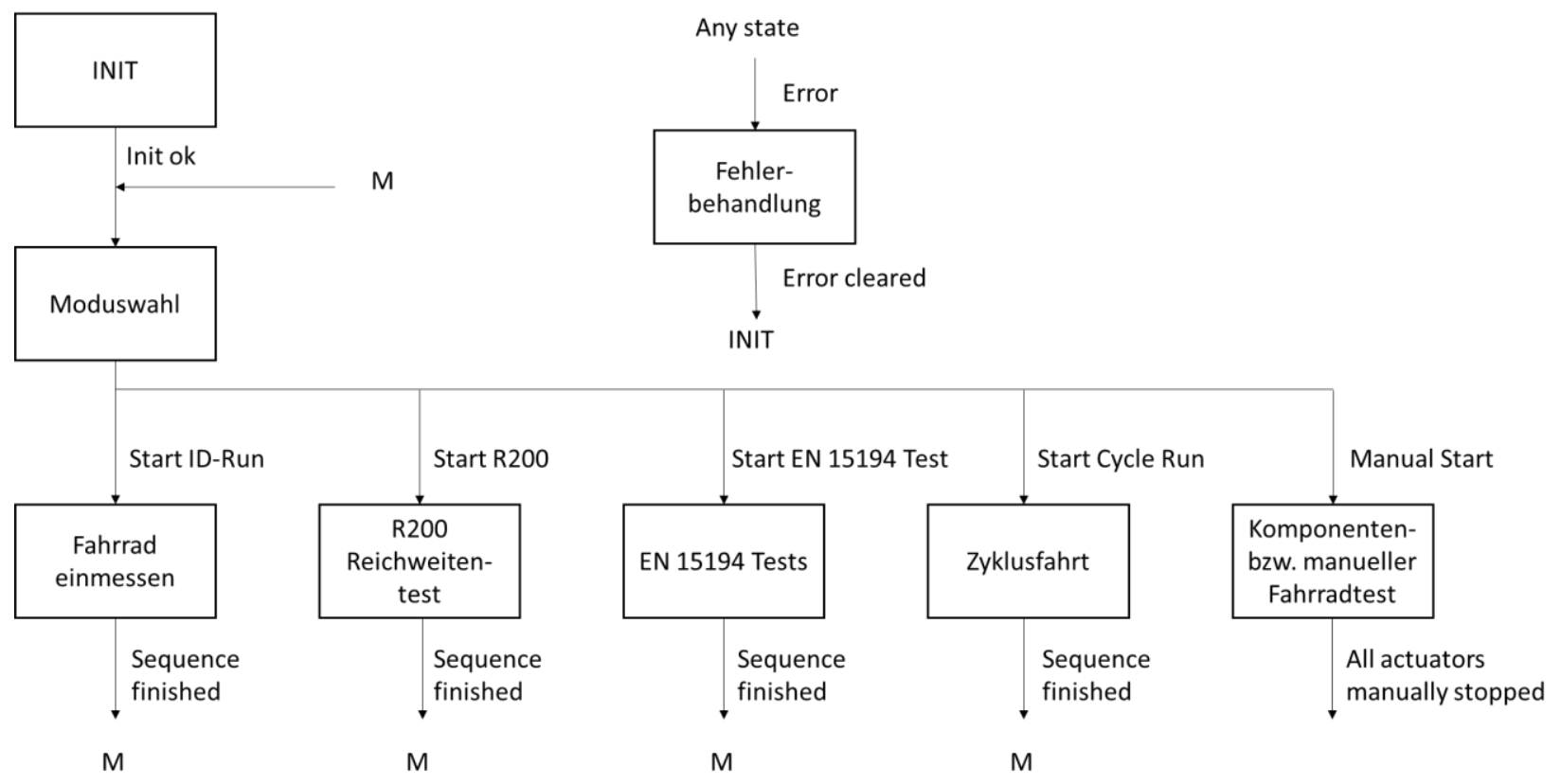

Bild 2: Main State Machine

Nach Initialisierung aller nötigen Parameter und Plausibilisierung der initialen Eingangssignale befindet sich die Anlage im Betriebszustand „Moduswahl“, von dem aus zu den einzelnen Betriebsarten verzweigt wird. In den automatischen Betriebszuständen „Fahrrad einmessen“, „R200 ReichweitenTest“ und „EN 15194 Tests“ laufen Schrittketten ab, die die automatisierten Abläufe steuern. Über den Zustand „Zyklusfahrt“ können generierte oder gemessene Fahrzyklen abgefahren werden. Schließlich können in den Zuständen „Komponententest“ bzw. „Manueller Fahrradtest“ wie oben beschrieben manuelle, d.h. nicht automatisierte, Tests gemacht werden. Das Ende eines automatischen Ablaufs bzw. das manuelle Stoppen aktiver Motoren führt einen wieder zur Moduswahl.

Ein Fehler hingegen führt in den Zustand „Fehlerbehandlung“. Dieser wird nach erfolgreicher Rücksetzung des Fehlers wieder in den „Init“-Zustand verlassen, um sicherzugehen, dass der Prüfstand wieder sauber startet. 


\section{Software-Struktur}

Die Software befindet sich aktuell noch in der Entwicklung. Zurzeit wird erwartet, dass ein einzelner Task zur Steuerung des Prüfstandes genügen wird.

Das Programm der Prüfstandsteuerung soll den charakteristischen Ablauf einer Speicherprogrammierbaren Steuerung (SPS) nach dem sogenannten „EVA-Prinzip“ aufweisen. Die Abkürzung „EVA“ gilt stellvertretend für die Wortlaute Eingabe, Verarbeitung und Ausgabe. Im Programmprozess wird kontinuierlich nach diesem Ablauf verfahren.

Entsprechend diesem Prinzip wird ein Programm (PRG) Input verwendet, das alle Eingangsvariablen von den Eingangsklemmen liest und in physikalische Werte konvertiert. Entsprechend gibt es ein PRG Output, das nach der Verarbeitung die Ausgangswerte in Feldbuswerte (Integer) skaliert und die Aktoren ansteuert.

Die Programmstruktur gliedert sich in die folgenden Hauptprogramme, s. Bild 3:

- Input (HW-Eingänge einlesen und umrechnen)

- Main State Machine (Zustandsautomat, Bestimmung Betriebszustand, Aufruf der entsprechenden Unterprogramme)

- Actuator Control (Funktionen zur Ansteuerung der einzelnen Aktoren, z.B. Freigaben für die Motoren, Regler etc.)

- Output (Variablen umrechnen und an die Ausgänge geben)

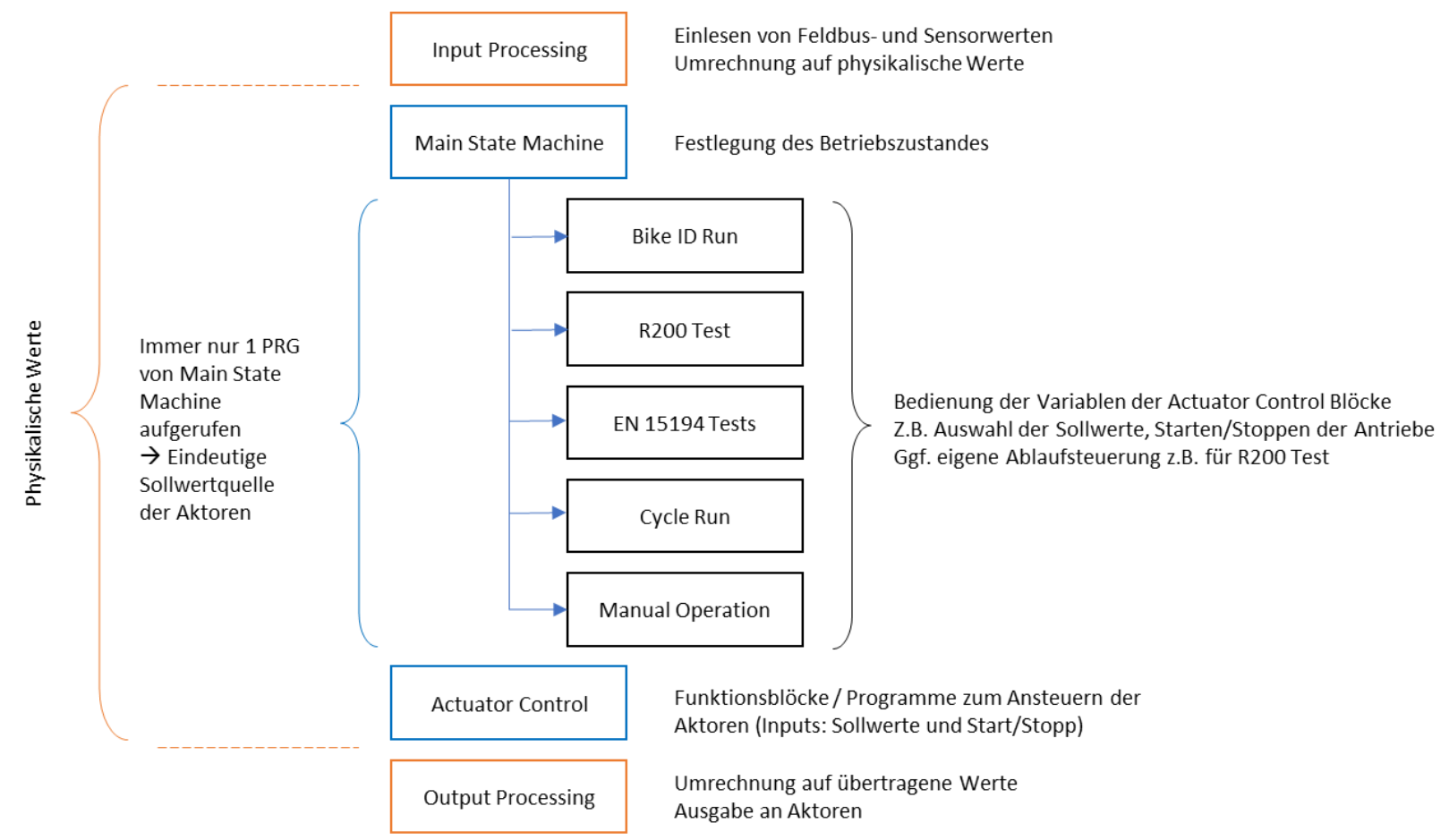

Bild 3: Programmstruktur

Durch dieses Konzept ist auch sichergestellt, dass es zu keinen Konflikten der Aktoransteuerung kommt. Die Sollwerte werden in den Unterprogrammen generiert, von denen immer nur eines von der State Machine aufgerufen wird. Das PRG Actuator Control nimmt die Sollwerte entgegen und steuert die Aktoren an. D.h. es gibt nur einen kontrollierenden Funktionsbaustein je Aktor. 


\section{Einbindung in die Lehre}

Schwerpunkt der aktuellen studentischen Arbeiten ist die Softwareseite. Studierende lernen in Studien-, Projekt- und Abschlussarbeiten die Programmierung einer bzw. Automatisierung mittels einer SPS kennen. Die aktuellen entsprechenden Arbeiten beschäftigen sich mit der generellen Programmstruktur, der Kommunikation zwischen den Programmen und den Datenstrukturen sowie der Implementierung der State Machine. Anschließend können die einzelnen Abläufe (R200 Test, Normtest etc.) implementiert werden.

Auch regelungs- und messtechnische Fragestellungen lassen sich anhand des Prüfstandes praktisch untersuchen. So beschäftigten sich vergangene Arbeiten mit der Robustheit der Linearaktor-Regler gegenüber Messfehlern.

Im Rahmen des Masterstudiengangs „Mechatronik“ ist ein zweisemestriges „Mechatronisches Projekt“ im Curriculum vorgesehen. Studierende arbeiten in Zweier- oder Dreierteams an einer gestellten Aufgabe. Hier ermöglicht der Pedelec-Prüfstand als mechatronisches System viele interessante Aufgaben zur praktischen Bereicherung des Studiums. So kann eine Projektgruppe im Laufe der zwei Semester beispielsweise Erfahrung in der SPS-Programmierung sammeln, Kennlinien der pneumatischen Aktoren mit Messungen abgleichen und mechanische Erweiterungen des Prüfstandes konstruieren.

Da insbesondere auf der Softwareseite noch viel zu tun ist, wird das Projekt „Pedelec-Prüstand“ sicherlich noch für einige Semester praxisnahe Aufgaben für studentische Arbeiten und Projekte bieten.

\section{Literatur}

[1] DIN EN 15194. Fahrräder - Elektromotorisch unterstützte Räder - EPAC. EN 15194:2017, 2017

[2] Statista: Absatz von E-Bikes in Deutschland von 2010 bis 2020. Online verfügbar unter https://de.statista.com/statistik/daten/studie/152721/umfrage/absatz-von-e-bikes-in-deutschland/, 2021

[3] Zweirad-Industrieverband (ZIV): Normierte Reichweite R200 für E-Bikes, 2018 